\title{
Structural relaxation in pure liquids: Analysis of wavenumber dependence within the approach of generalized collective modes
}

\author{
T.Bryk $^{1,2}$, I.Mryglod ${ }^{1,2}$ \\ 1 Institute for Condensed Matter Physics, National Academy of Sciences of Ukraine, \\ 1 Svientsitskii St., 79011 Lviv, Ukraine \\ 2 Institute of Applied Mathematics and Fundamental Sciences \\ Lviv Polytechnic National University, 12 Bandery Str., 79013 Lviv, Ukraine
}

Received January 4, 2008, in final form February 28, 2008

\begin{abstract}
Wavenumber dependence of structural relaxation in liquids is studied by the method of generalized collective modes (GCM). A new perturbation approach within the GCM method is proposed and applied in the longwavelenth limit in order to obtain analytical expressions for the wavenumber-dependent structural relaxation and sound dispersion within a simplified three-variable dynamical model. Analytical results are compared with numerical study of generalized modes within a more general five-variable dynamical model, which accounts for thermal processes in liquids. Numerical results are presented for four thermodynamic points of LennardJones fluid at the reduced temperature $T^{*}=1.71$. We discuss the features of non-hydrodynamic process of structural relaxation in different regions of wavenumbers.
\end{abstract}

Key words: generalized hydrodynamics, structural relaxation, dynamical models, sound excitations, positive dispersion

PACS: 05.20.Jj, 61.20.Lc, 62.60.+v

\section{Introduction}

Collective dynamics in liquids is so far well understood mainly on macroscopic length and time scales, where the hydrodynamic theory (i.e. local conservation laws) is applicable [1,2]. On the spatial scales comparable with mean interatomic distance the microscopic dynamics in liquids is essentially more rich than in hydrodynamic region: there exist non-hydrodynamic collective processes with finite lifetime, which contribute to all measurable quantities. Examples of non-hydrodynamic propagating processes are shear waves and heat waves (zero-sound), charge waves in ionic liquids, while most obvious non-hydrodynamic relaxation process is the structural relaxation. In contrast to hydrodynamic processes the non-hydrodynamic ones do not survive on macroscopic distances and times due to finite lifetime or inability of liquids to support the specific propagating process on macroscopic length scales as this is in case of shear waves. The non-hydrodynamic processes, sometimes yet called as kinetic ones [3], should have wavenumber dependence of their specific lifetimes $\tau_{i}(k)$, as well as frequencies $\omega_{i}(k)$ for propagating kinetic modes, essentially different from hydrodynamic ones.

The standard inverse lifetimes for collective processes obtained within the hydrodynamic description are proportional to $k^{2}$. For pure liquids there exist two hydrodynamic collective relaxation processes: thermal and viscous relaxations with inverse lifetimes $\tau_{\text {th }}^{-1}(k)=\lambda k^{2} / n c_{\mathrm{V}}$ and $\tau_{\mathrm{v}}^{-1}(k)=\eta k^{2} / \rho$, respectively, with $\lambda, \eta, c_{\mathrm{V}}$ being macroscopic thermal conductivity, shear viscosity and specific heat at constant volume. Herein $\rho$ and $n$ denote mass and numerical densities of the system, respectively. The single hydrodynamic propagating process, which corresponds to longitudinal acoustic excitations, has the inverse lifetime $\Gamma k^{2}$, which essentially is dependent on a 
coupling between viscous and thermal processes. Here the sound attenuation coefficient

$$
\Gamma=\frac{1}{2}\left[D_{\mathrm{L}}+(\gamma-1) D_{\mathrm{T}}\right]
$$

where the ratio of specific heats $\gamma=c_{\mathrm{P}} / c_{\mathrm{V}}$ is a measure of such coupling, $D_{\mathrm{L}}=\left(\frac{4}{3} \eta+\zeta\right) / \rho$ is kinematic viscosity, $\zeta$ - bulk viscosity, and $D_{\mathrm{T}}=\lambda / n c_{\mathrm{P}}$ is thermal diffusivity. For the case of $\nu$-component liquids there appear in addition other $\nu-1$ hydrodynamic relaxation processes connected with mutual diffusivity of species. The inverse lifetimes of hydrodynamic relaxation processes are connected with relevant transport coefficients. Another important point is that within the hydrodynamic description the relaxation processes enter to the density-density, density-energy and energy-energy time correlation functions as exponentially decaying with time terms, that simply implies definition of their specific lifetimes $\tau_{i}(k)[4]$.

Non-hydrodynamic processes contribute to all physical quantities, which can be measured on relevant spatial and temporal scales. Among them are the wavenumber- and frequency-dependent spectral functions $I(k, \omega)$ obtained in INS or IXS experiments, or time correlation functions $F_{i j}(k, t)$ calculated in molecular dynamics simulations. Outside the hydrodynamic region the nonhydrodynamic processes should essentially affect the shape of time correlation functions, and there appears a problem how to extract in a correct way the contributions of non-hydrodynamic processes from the calculated $F_{i j}(k, t)$ or measured intensities $I(k, \omega)$. There were many attempts to take into account the existence of non-hydrodynamic processes in fluids for analysis of scattering experiments [5-7]. But still the issue of non-hydrodynamic processes and their effect on the dispersion of the acoustic excitations is among the unsolved problems of the collective dynamics in liquids [8]. Perhaps the most obvious relaxation process, not reflected in hydrodynamic approach, is the structural relaxation, which is related to cooperative rearrangements of the local structure due to density fluctuations. Although the structural relaxation is one of the most studied relaxation processes for supercooled liquids and at the glass transition, its role and contribution to the time correlation functions in the liquid state is not well understood. Moreover, little is known about the wavenumber dependence of structural relaxation in liquids. One of the first studies of the wavenumber dependence of structural relaxation in liquids [9] resulted in conclusion, that in the long-wavelength limit the lifetime of structural relaxation must tend to a finite value.

The mostly used approach in the studies of effects of structural relaxation in liquids was based on a memory function formalism $[2,10]$ and appeared to be quite successful in explaining the temperature dependence of structural relaxation in liquids [11]. The approach itself was based on assumption, that the second-order memory function can be separated into different decay channels, each of which corresponded to a specific collective process: thermal relaxation, structural relaxation, instantaneous relaxation (for time decay of the memory function on time scales much faster than the experimentally accessed frequency window), etc. Relaxation processes entered the second-order memory function as exponentially decaying in time terms, while instantaneous decay channel was represented via time delta-function $[12,13]$. Relaxation times of the decay channels (corresponding factors in exponentials of the second-order memory function) were treated as fitting parameters. The generalized in this way expression for the dynamical structure factor $S(k, \omega)$ after convolution with a measured instrumental resolution function was used in fitting to experimental scattering intensities in wide region of wavenumbers and resulted in $k$-dependent relaxation time of structural relaxation. Such an approach resulted in very reasonable Arrhenius-type behaviour of temperature dependence of extrapolated to long-wavelength limit of structural relaxation $\tau_{\text {str }}(T)$ $[14,15]$. However, it is not clear yet, whether the relaxation times associated with the decay channels of the second-order memory function can be directly related in the whole range of wavenumbers to relaxation times of physical processes. It was demonstrated in [16], that the hydrodynamic form of dynamical structure factor can be reproduced by a suitable choice of two decay channels of the second-order memory function. However, there does not exist a proof of correct wavenumber dependence of relaxation time of structural relaxation, obtained within the fitting procedure with additional decay channels in a wide region of wavenumbers.

There exists another theoretical method, known as the approach of Generalized Collective Modes (GCM), which is closely related to the memory function formalism. However, the essential 
difference of the GCM approach is treatment of non-hydrodynamic processes via extended set of hydrodynamic variables and orthogonal to them short-time dynamical variables, which is applied for solving of the generalized Langevin equation in terms of dynamical eigenmodes. The GCM approach treats hydrodynamic and non-hydrodynamic processes on the same footing and takes into account local coupling effects between them. All the relaxation processes - hydrodynamic and kinetic ones - contribute directly to the shape of all time correlation functions of interest via exponentially decaying in time terms. Hence, the GCM approach can shed light on the problem of wavenumber dependence of structural relaxation in liquids, because it is straightforward by analogy with hydrodynamic relaxation processes to associate particular exponential term (with real eigenvalue) in the relevant time correlation function with the non-hydrodynamic process of structural relaxation, and it is obvious that the corresponding real eigenvalue will define the inverse wavenumber-dependent specific time of structural relaxation $d_{\mathrm{str}}(k)=\tau_{\mathrm{str}}^{-1}(k)$.

The remaining paper is organized as follows. In the next Section we describe the approach of Generalized Collective Modes for analysis of collective dynamics in liquids. In Section 3 analytical solutions for a simplified three-variable model are presented in order to estimate the longwavelength asymptote of relaxation mode connected with structural relaxation. Section 4 contains results of MD simulations and numerical GCM analysis of relaxation processes in a wide range of wavenumbers, and the last Section contains conclusions of this study.

\section{The approach of generalized collective modes}

The GCM approach consists in solving the generalized Langevin equation, which is represented in matrix form generated on a chosen set of dynamical variables, in terms of eigenmodes and their contributions to the matrix of time correlation functions. The most simple formulation of the GCM approach is based on Markovian approximation for the highest-order memory functions, although this is not the limitation and a more sophisticated version of the GCM approach is available [17]. Usually the basis set of $N_{\mathrm{v}}$ dynamical variables is constructed by extension of the set of hydrodynamic variables, which describe fluctuations of conserved quantities, by their time derivatives, which being orthogonal to hydrodynamic slow fluctuations are aimed to describe correctly more short-time fluctuations, which are not considered within hydrodynamic theory. For pure liquids the hydrodynamic set of variables for description of longitudinal dynamics consists of three dynamical variables:

$$
\mathbf{A}^{(3 \mathrm{hyd})}(k, t)=\left\{n(k, t), J^{L}(k, t), e(k, t)\right\},
$$

where

$$
n(k, t)=\frac{1}{\sqrt{N}} \sum_{i=1}^{N} \mathrm{e}^{\mathrm{i} \mathbf{k r}_{i}(t)},
$$

is the $k$-th spatial-Fourier component of particle density of the system composed of $N$ particles with instantaneous positions $\mathbf{r}_{i}(t)$ and velocities $\mathbf{v}_{i}(t)$,

$$
J^{L}(k, t)=\frac{m}{\sqrt{N}} \frac{1}{k} \sum_{i=1}^{N} \mathbf{k v}_{i} \mathrm{e}^{\mathrm{ikr} \mathbf{r}_{i}(t)}
$$

is the Fourier-component of longitudinal part of mass-current density, and the energy density is

$$
e(k, t)=\frac{1}{\sqrt{N}} \sum_{i=1}^{N} \varepsilon_{i} \mathrm{e}^{\mathrm{i} \mathbf{k r}_{i}(t)},
$$

where $\varepsilon_{i}$ is the single-particle energy of $i$-th particle. The dynamical variables (2)-(4) correspond to fluctuations of conserved quantities. One can restrict the treatment of dynamical processes just by a set of equations with only dynamical variables of fluctuating conserved quantities and 
in Markovian approximation this results to expressions valid on macroscopic distances and time scales only.

In order to account for non-hydrodynamic processes within the GCM approach one applies for construction of all matrices an extended set of dynamical variables

$$
\mathbf{A}^{\left(N_{\mathrm{v}}\right)}(k, t)=\mathbf{A}^{(3 \mathrm{hyd})}(k, t) \oplus\left\{\dot{J}^{L}(k, t), \dot{e}(k, t), \ddot{J}^{L}(k, t), \ddot{e}(k, t), \ldots\right\},
$$

where overdots mean the corresponding order of time derivatives. The chosen set of $N_{\mathrm{v}}$ dynamical variables is used for construction of the matrix of static correlation functions $\mathbf{F}(k, t=0)$ with elements

$$
F_{i j}(k, t=0)=\left\langle A_{i}^{*}(k, t=0) A_{j}(k, t=0)\right\rangle,
$$

where the asterisk means complex conjugation, and matrix of corresponding Laplace-transformed time correlation functions $\tilde{\mathbf{F}}(k, z)$. In the simplest Markovian approximation the matrix form of generalized Langevin equation for Laplace-components of time correlation functions reads

$$
\tilde{\mathbf{F}}(k, z)=[z \mathbf{I}+\mathbf{T}(k)]^{-1} \mathbf{F}(k, t=0)
$$

with $\mathbf{T}(k)$ called as generalized hydrodynamic matrix and expressed via

$$
\mathbf{T}(k)=-\mathbf{i} \boldsymbol{\Omega}(k)+\tilde{\mathbf{M}}(k, z=0) \equiv \mathbf{F}(k, t=0) \tilde{\mathbf{F}}^{-1}(k, z=0)
$$

Here $\boldsymbol{\Omega}(k)$ and $\tilde{\mathbf{M}}(k, z)$ are the standard frequency matrix and matrix of memory functions, respectively. The number of successive time derivatives of some hydrodynamic variable in the basis set defines the order of the highest memory function, which is taken in Markovian approximation. We stress, that using a large number of successive time derivatives of hydrodynamic variables (large number of dynamical variables $N_{\mathrm{v}}$ ) one applies the Markovian approximation to the very high-order memory function, that is quite justified, because the high-order memory functions are rapidly decaying functions of time.

The generalized hydrodynamic matrix can be diagonalized using the solutions of eigenvalue problem

$$
\sum_{j=1}^{N_{\mathrm{v}}} T_{i j}(k) X_{j}^{\alpha}(k)=z_{\alpha}(k) X_{i}^{\alpha}(k),
$$

where $z_{\alpha}(k)$ and $X_{i}^{\alpha}(k)$ are the $\alpha$-th eigenvalue and corresponding eigenvector, respectively. In terms of the eigenvalues and eigenvectors the solution of generalized Langevin equation is represented as follows:

$$
\tilde{F}_{i j}(k, z)=\sum_{\alpha=1}^{N_{\mathrm{v}}} \frac{G_{i j}^{\alpha}(k)}{z+z_{\alpha}(k)},
$$

which transforming back to time dependence yields

$$
F_{i j}(k, t)=\sum_{\alpha=1}^{N_{\mathrm{v}}} G_{i j}^{\alpha}(k) \mathrm{e}^{-z_{\alpha}(k) t} .
$$

The weight coefficients $G_{i j}^{\alpha}(k)$ are simply estimated from the relevant eigenvectors [18]. It is important, that the time correlation functions are represented as a sum of $N_{\mathrm{v}}$ separated contributions from different dynamical eigenmodes. The $N_{\mathrm{v}}$ eigenvalues $z_{\alpha}(k)$ of the matrix $\mathbf{T}(k)$ correspond either to propagating modes (complex-conjugated pairs of eigenvalues) or to relaxation processes (real eigenvalues), which contribute with the strength $G_{i j}^{\alpha}(k)$ to the shape of relevant time correlation function $F_{i j}(k, t)$ as oscillating or exponential functions of time, respectively. For convenience we will further note the purely real eigenvalues as $d_{\alpha}(k)$.

The number of successive time derivatives of hydrodynamic variables in the basis set defines the order of frequency sum rules fulfilled for the solutions $\tilde{F}_{i j}(k, z)$. In general the presence of $s$ successive time derivatives of hydrodynamic variable $A_{i}(k, t)$ in the basis set results in $2 s+1$ 
frequency sum rules fulfilled for the theoretical representation (GCM replica) of relevant $\tilde{F}_{i i}(k, z)$. Several additional advantages of the GCM approach over the traditional memory function method are the following: (i) an additional sum rule which requires the equivalence of relevant correlation time with some reference correlation time allows to avoid a priori modeling of memory functions. The reference correlation time either in analytical form in hydrodynamic regime, or it can be obtained numerically for a given system as a function of wavenumbers from MD simulations; (ii) the time correlation functions (and relevant dynamical structure factors) are expressed as a separable sum over $N_{\mathrm{v}}$ mode contributions from extended hydrodynamic and non-hydrodynamic modes in the whole range of wavenumbers in analogy as this is in hydrodynamic theory. Each collective excitation is characterized by a complex eigenvalue $z_{\alpha}(k)$ and corresponding amplitude of mode contribution $G_{i j}^{\alpha}(k)$ to the shape of dynamical structure factor $S_{i j}(k, \omega)$.

\section{Analytical treatment in long-wavelength region via perturbation approach}

One of the simplest dynamical models, which permits us to find analytical expressions and to describe a relaxation kinetic process of non-thermal origin is a three-variable dynamical model with the following dynamical variables:

$$
\mathbf{A}^{(3)}(k, t)=\left\{n(k, t), J^{L}(k, t), \dot{J}^{L}(k, t)\right\}
$$

where the first time derivative of longitudinal component of mass-current is

$$
\dot{J}^{L}(k, t)=\frac{1}{\sqrt{N}} \frac{1}{k} \sum_{i=1}^{N}\left[\left(\mathbf{k F}_{i}\right)+\mathrm{i}\left(\mathbf{k v}_{i}\right)^{2}\right] \mathrm{e}^{\mathrm{i} \mathbf{k r} \mathbf{r}_{i}(t)} .
$$

Here $\mathbf{F}_{i}(t)$ denotes the force acting on the $i$-th particle. The only non-hydrodynamic variable among these three ones is $\mathbf{j}(k, t)$ and it is connected to Fourier-components of stress tensor $\sigma_{\alpha \beta}(k, t)$ via relation $[5]$ :

$$
\frac{\mathrm{d}}{\mathrm{d} t} \mathbf{J}(k, t)=\mathrm{i} \mathbf{k} \hat{\sigma}(k, t),
$$

i.e. in the generalized hydrodynamic description appear quantities from theory of elasticity.

It is quite straightforward to construct the $3 \times 3$ generalized hydrodynamic matrix on the basis set of non-orthogonal dynamical variables (12) and find its eigenvalues. However, to make the results and origin of eigenmodes more transparent we will apply a perturbation approach recently elaborated within the GCM method. The idea is to separate in the generalized hydrodynamic matrix $\mathbf{T}(k)$ a part, $\mathbf{T}_{\mathbf{0}}(k)$, which permits simple analytical solution in terms of "bare" modes [19], while the remaining part $\delta \mathbf{T}(k)$ contains local coupling effects between "bare" modes and is treated as perturbation. If the perturbation $\delta \mathbf{T}(k)$ is essentially smaller than the distance between "bare" modes, one can expect the rapid convergence of perturbation series.

First of all in order to simplify perturbation series one has to construct the generalized hydrodynamic matrix $\mathbf{T}(k)$ on orthonormal basis set of dynamical variables. Therefore we can transform initial basis set (12) as follows:

$$
\left(\begin{array}{c}
\tilde{n}_{k} \\
\tilde{J}_{k}^{L} \\
\tilde{J}_{k}^{L}
\end{array}\right)=\mathbf{L}\left(\begin{array}{c}
n_{k} \\
J_{k}^{L} \\
\dot{J}_{k}^{L}
\end{array}\right)
$$

where the matrix of linear transformation

$$
\mathbf{L}(k)=\left(\begin{array}{ccc}
\frac{1}{\sqrt{S(k)}} & 0 & 0 \\
0 & \frac{1}{\sqrt{m k_{\mathrm{B}} T}} & 0 \\
-\mathrm{i} k \frac{m c_{\mathrm{T}}}{\sqrt{\omega_{1}^{2}(k)-k^{2} c_{\mathrm{T}}^{2}(k)}} & 0 & \frac{1}{\sqrt{\omega_{1}^{2}(k)-k^{2} c_{\mathrm{T}}^{2}(k)}}
\end{array}\right) .
$$


Here

$$
c_{\mathrm{T}}^{2}(k)=\frac{k_{\mathrm{B}} T}{m S(k)}
$$

is the square of isothermal velocity, and

$$
\omega_{\mathrm{l}}^{2}(k)=\frac{\left\langle\dot{J}_{-k}^{L} \dot{J}_{k}^{L}\right\rangle}{\left\langle J_{-k}^{L} J_{k}^{L}\right\rangle} \stackrel{k \rightarrow 0}{\rightarrow} c_{\infty}^{2} k^{2},
$$

where $c_{\infty}$ is the high-frequency speed of sound. Now the tilded variables in (14) satisfy the condition

$$
\left\langle\tilde{a}_{-k} \tilde{b}_{k}\right\rangle=\delta_{a b}
$$

The generalized hydrodynamic matrix $\mathbf{T}(k)$, constructed on the set of non-orthogonal variables can be simply related via linear transformation with the matrix $\tilde{\mathbf{T}}(k)$, defined on the orthogonalized variables

$$
\tilde{\mathbf{T}}(k)=\mathbf{L T L}^{-\mathbf{1}} .
$$

Obviously the eigenvalues of both matrices $\mathbf{T}(k)$ and $\tilde{\mathbf{T}}(k)$ are the same. Hereby, one has to find eigensolutions of the generalized hydrodynamic matrix

$$
\tilde{\mathbf{T}}(k)=\left(\begin{array}{ccc}
0 & -\mathrm{i} k c_{\mathrm{T}} & 0 \\
-\mathrm{i} k c_{\mathrm{T}} & 0 & -\sqrt{\omega_{1}^{2}(k)-k^{2} c_{\mathrm{T}}^{2}} \\
0 & \sqrt{\omega_{1}^{2}(k)-k^{2} c_{\mathrm{T}}^{2}} & \left(\frac{\omega_{1}^{2}(k)}{k^{2} c_{\mathrm{T}}^{2}}-1\right) \frac{1}{\tau(k)}
\end{array}\right),
$$

where the quantity $\tau(k)$ is the correlation time associated with the density-density correlations without coupling to thermal processes. In general one does not know the wavenumber dependence of $\tau(k)$ in the whole range of wavenumbers, therefore within the GCM approach one is required to use molecular dynamics simulations in order to have numerical dependence of $\tau(k)$ as input quantity. In case of dynamical models with explicit treatment of density and thermal fluctuations one calculates the generalized correlation times via their definition

$$
\tau_{i j}(k)=\frac{1}{F_{i j}(k, t=0)} \int_{0}^{\infty} F_{i j}(k, t) \mathrm{d} t,
$$

where $F_{i j}(k, t)$ are the density-density, density-energy or energy-energy time correlation functions calculated in MD simulations for given wavenumber $k$.

The density fluctuations in liquids on macroscopic scale are always constrained to local law of energy conservation. The three-variable model (12) considered here, however, does not take implicitly into account coupling between energy and density fluctuations, therefore the correlation time $\tau(k)$ cannot be taken from MD simulations - the density-density time correlation functions for any $k$ value always have contribution from thermal processes, although for intermediate and large wavenumbers that contribution is quite small and can be neglected in simplest approximations. However, in analytical approaches it is possible to remove the contribution from thermal processes - this is made by setting $\gamma=1$. Therefore, we can use analytical expression for density-density time correlation functions [4] in the long-wavelength limit and obtain the correlation time in viscoelastic approximation:

$$
\tau(k) \stackrel{k \rightarrow 0}{\rightarrow} \frac{D_{\mathrm{L}}}{c_{\mathrm{T}}^{2}}
$$

We stress, that in contrast to the hydrodynamic correlation times (19) with long-wavelength asymptotes $k^{-2}$ the correlation time $\tau(k)$ in viscoelastic theory (i.e. idealized model without coupling to thermal fluctuations) tends to a constant in $k \rightarrow 0$ limit. This is because all three hydrodynamic time correlation functions of longitudinal dynamics in pure liquids [4] have similar exponential contributions of the same origin, which come from thermal relaxation. 
We proceed further with separating the generalized hydrodynamic matrix $\tilde{\mathbf{T}}(k)$ into two parts: $\tilde{\mathbf{T}}_{\mathbf{0}}(k)+\delta \tilde{\mathbf{T}}(k)$. The first matrix

$$
\tilde{\mathbf{T}}_{0}(k)=\left(\begin{array}{ccc}
0 & -\mathrm{i} k c_{\mathrm{T}} & 0 \\
-\mathrm{i} k c_{\mathrm{T}} & 0 & 0 \\
0 & 0 & \left(\frac{\omega_{1}^{2}}{k^{2} c_{\mathrm{T}}^{2}}-1\right) \frac{1}{\tau}
\end{array}\right)
$$

can be immediately analytically solved in terms of eigenvalues and eigenvectors in the whole region of wavenumbers. Among the eigenvalues in the long-wavelength limit there are a single real number

$$
d^{(0)}(k) \equiv d_{0}=\frac{c_{\infty}^{2}-c_{\mathrm{T}}^{2}}{c_{\mathrm{T}}^{2}} \frac{1}{\tau}
$$

and a pair of conjugated imaginary numbers

$$
z_{ \pm}^{(0)}(k)= \pm \mathrm{i} c_{\mathrm{T}} k
$$

Note, that in this case purely imaginary eigenvalues correspond to propagating waves without any damping, while purely real eigenvalues $d_{i}(k)$ are associated with relaxation process with specific relaxation time $\tau_{i}(k)=d_{i}^{-1}(k)$. The eigenvalues (22) and (23) can be treated as "bare" modes, which due to coupling between propagating and relaxation processes, considered further as a perturbation, will obtain $k$-dependent corrections. The eigenvectors associated with relevant "bare" modes are:

$$
\psi_{d}^{(0)}=\left(\begin{array}{l}
0 \\
0 \\
1
\end{array}\right), \quad \psi_{ \pm}^{(0)}=\frac{1}{\sqrt{2}}\left(\begin{array}{c}
\mp 1 \\
1 \\
0
\end{array}\right) .
$$

The first order corrections to both kinds of "bare" modes

$$
\delta z_{ \pm}^{(1)}(k)=\psi_{ \pm}^{(0) *} \delta \tilde{\mathbf{T}} \psi_{ \pm}^{(0)}=0
$$

and

$$
\delta d^{(1)}(k)=\psi_{d}^{(0) *} \delta \tilde{\mathbf{T}} \psi_{d}^{(0)}=0
$$

are equal to zero. Here the matrix of perturbation is

$$
\delta \tilde{\mathbf{T}}(k)=\left(\begin{array}{ccc}
0 & 0 & 0 \\
0 & 0 & -\sqrt{\omega_{1}^{2}-k^{2} c_{\mathrm{T}}^{2}} \\
0 & \sqrt{\omega_{1}^{2}-k^{2} c_{\mathrm{T}}^{2}} & 0
\end{array}\right),
$$

and in long-wavelength limit it is linear in $k$. The absence of first-order corrections to the eigenvalues means, that there are no linear in $k$ corrections to the "bare" modes. The first nonzero correction to the "bare" modes appears in the second order with respect to perturbation. Thus by keeping the leading in $k$ term one has

$$
\delta d^{(2)}(k)=\sum_{ \pm} \frac{\left(\psi_{d}^{(0) *} \delta \tilde{\mathbf{T}} \psi_{ \pm}^{(0)}\right)\left(\psi_{ \pm}^{(0) *} \delta \tilde{\mathbf{T}} \psi_{d}^{(0)}\right)}{d^{(0)}-z_{ \pm}^{(0)}}=-c_{\mathrm{T}}^{2} t k^{2},
$$

and this correction is proportional to $k^{2}$. Using (20) one obtains

$$
d(k)=\frac{c_{\infty}^{2}-c_{\mathrm{T}}^{2}}{D_{\mathrm{L}}}-D_{\mathrm{L}} k^{2} .
$$

The negative sign of the second order correction means, that with increasing wavenumbers the lifetime of kinetic relaxation process increases. The factor $\left(c_{\infty}^{2}-c_{\mathrm{T}}^{2}\right)$ is associated in the literature 
with the "strength" of structural relaxation [15], that implies the relevance of the real dynamical eigenmode $d(k)$ in $(27)$ with the structural relaxation.

For propagating modes one obtains

$$
\delta z_{ \pm}^{(2)}(k)=\frac{\left(\psi_{ \pm}^{(0) *} \delta \tilde{\mathbf{T}} \psi_{d}^{(0)}\right)\left(\psi_{d}^{(0) *} \delta \tilde{\mathbf{T}} \psi_{ \pm}^{(0)}\right)}{z_{ \pm}^{(0)}-d^{(0)}}=\frac{\left(c_{\infty}^{2}-c_{\mathrm{T}}^{2}\right) k^{2}\left(z_{ \pm}^{(0)}+d^{(0)}\right)}{c_{\mathrm{T}}^{2} k^{2}+d_{0}^{2}}
$$

that results in

$$
z_{ \pm}(k)=\frac{D_{\mathrm{L}}}{2} k^{2} \pm \mathrm{i} c_{\mathrm{T}} k\left(1+\frac{D_{\mathrm{L}}}{d_{0}} k^{2}\right)
$$

It follows, that due to coupling with the relaxation process $d(k)$ the "bare" propagating modes obtain damping proportional to $k^{2}$ and a correction to dispersion law, which can be treated as a positive deviation from linear dispersion. This positive dispersion is proportional to the kinematic viscosity and to the long-wavelength limit of structural relaxation time.

For eigenvectors the first-order corrections can be written as follows

$$
\delta \psi_{d}^{(1)}=-\frac{\sqrt{\omega_{1}^{2}-k^{2} c_{\mathrm{T}}^{2}}}{d_{0}^{2}+k^{2} c_{\mathrm{T}}^{2}}\left(\begin{array}{c}
-\mathrm{i} k c_{\mathrm{T}} \\
d_{0} \\
0
\end{array}\right), \quad \delta \psi_{ \pm}^{(1)}=-\frac{\left(d_{0} \pm \mathrm{i} k c_{\mathrm{T}}\right) \sqrt{\omega_{1}^{2}-k^{2} c_{\mathrm{T}}^{2}}}{\sqrt{2}\left(d_{0}^{2}+k^{2} c_{\mathrm{T}}^{2}\right)}\left(\begin{array}{l}
0 \\
0 \\
1
\end{array}\right)
$$

Using the properly normalized eigenvectors within the first order in perturbation $\psi_{d}$ and $\psi_{ \pm}$one can estimate the contributions from relaxation and propagating processes to all time correlation functions and corresponding spectral functions of interest. For the density-density time correlation function the model (12) results in three contributions with purely real weight coefficients from propagating modes $B_{i j}(k)$ and $D_{i j}(k)$ :

$$
\frac{F_{n n}(k, t)}{S(k)}=A_{n n}(k) \mathrm{e}^{-d(k) t}+\left(B_{n n} \cos \omega(k) t+D_{n n} \sin \omega(k) t\right) \mathrm{e}^{-\sigma(k) t}
$$

where frequency $\omega(k)$ and damping $\sigma(k)$ of propagating modes are obtained from $z_{ \pm}(k, t)$ as imaginary and real parts, respectively. We are mainly interested to look at the long-wavelength asymptote of contribution from kinetic relaxation process to the density-density time correlation function $A_{n n}(k \rightarrow 0)$. This weight coefficient can be obtained from the corresponding eigenvectors simply as follows:

$$
A_{n n}(k)=\psi_{d, 1} \psi_{d, 1}^{*}
$$

that results in the following asymptotic value

$$
A_{n n}(k \rightarrow 0)=\frac{c_{\mathrm{T}}^{2} D_{\mathrm{L}}}{d_{0}^{3}} k^{4}
$$

Completely in agreement with the concept of kinetic collective processes the contribution from the structural relaxation to the density-density time correlation function vanishes by approaching the hydrodynamic region. The special feature of structural relaxation is that the relaxation time for this mode is finite in the hydrodynamic limit and its contribution decays even faster than $k^{2}$ when $k$ is small.

The presented perturbation approach applied within the GCM scheme can be very effective for finding analytical solutions of more sophisticated dynamical models. It is problematic to solve analytically the eigenvalue problem for many-variable dynamical models even in the long-wavelength limit. Therefore the presented GCM perturbation approach is very promising for analytical studies of collective dynamics of binary liquids, disparate mass two-component systems and three-component liquid mixtures, for which only simplest analytical solutions exist to the date [19-21]. 


\section{Numerical GCM analysis of collective dynamics: Lennard-Jones fluids}

For the study of collective modes of dense liquids in a wide region of wavenumbers one has to apply within the GCM approach more sophisticated basis sets of dynamical variables that take into account the main non-hydrodynamic processes within the same level of accuracy. The simplest set of dynamical variables within the precision of the fourth sum rule for the density-density time correlation function is the five-variable set

$$
\mathbf{A}^{(5)}(k, t)=\left\{n(k, t), J^{L}(k, t), e(k, t), \dot{J}^{L}(k, t), \dot{e}(k, t)\right\} .
$$

This five-variable dynamical model permits also an analytical GCM solution for collective modes in long-wavelength limit [22], that makes possible to compare the expressions obtained in the previous Section within the simplified three-variable dynamical model and perturbation approach. The dynamical model (34) is able to account for two types of non-hydrodynamic relaxation processes: structural relaxation mode $\left(d_{2}(k)\right.$ in notations of [22] in order to distinguish with the lowest hydrodynamic thermal process $\left.d_{\mathrm{T}}(k)=d_{1}(k)\right)$ and kinetic heat relaxation mode $\left(d_{3}(k)\right)$. The analytical expression for $d_{2}(k)$, obtained in the long-wavelength limit in [22] reads

$$
d_{2}(k)=d_{2}^{0}-D_{\mathrm{L}} k^{2}+(\gamma-1) \Delta k^{2},
$$

and can be easily transformed to the obtained above equation (27) by setting $\gamma=1$, i.e. neglecting coupling with heat fluctuations. In equation (35) the following notations were applied

$$
\Delta=\frac{d_{2}^{0} d_{3}^{0}}{d_{3}^{0}-d_{2}^{0}} \frac{D_{\mathrm{T}}}{D_{\mathrm{L}} c_{\mathrm{S}}^{2}}\left(D_{\mathrm{T}}-D_{\mathrm{L}}\right)^{2},
$$

where

$$
d_{2}^{0}=\frac{c_{\infty}^{2}-c_{\mathrm{s}}^{2}}{D_{\mathrm{L}}}, \quad d_{3}^{0}=\frac{c_{\mathrm{V}}}{m \lambda}\left(G^{\mathrm{h}}-\frac{\gamma-1}{\kappa_{\mathrm{T}}}\right),
$$

$G^{\mathrm{h}}$ and $\kappa_{\mathrm{T}}$ are the heat rigidity and isothermal compressibility of the liquid, respectively.

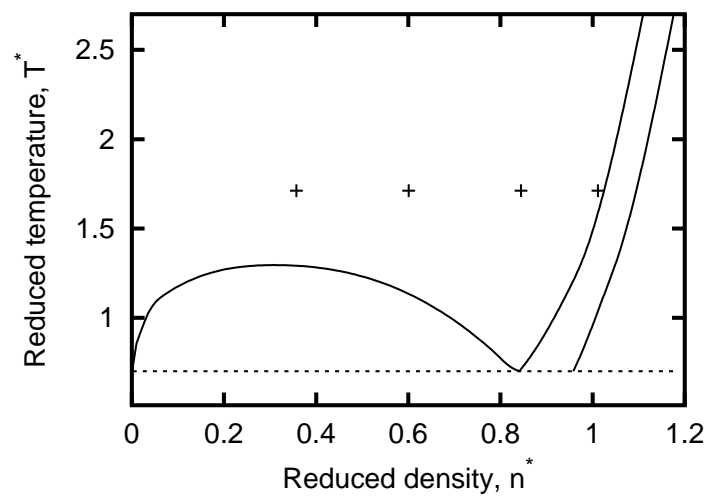

Figure 1. Thermodynamic points on the phase diagram of Lennard-Jones fluids along the isothermal line $T^{*}=1.71$ used in numerical GCM study.

Now we apply the five-variable dynamical model for numerical study of collective modes in Lennard-Jones fluids. We consider four thermodynamic points with different density, shown by symbols along the isothermal line $T^{*}=1.71$ on the phase diagram of Lennard-Jones fluids in figure 1. For these thermodynamic points we performed MD simulations with 2000 (for small wavenumbers) and 1000 particles in order to calculate density-density, energy-density and energyenergy time correlation functions in the wavenumber region up to $3 \AA^{-1}$. The majority production runs were performed over 300000 steps, while for small wavenumbers we simulated the systems over one million timesteps to obtain good convergence of relevant static averages needed in the 
GCM analysis. In figure 2 the MD-derived density-density, density-energy and energy-energy time correlation functions along with their theoretical GCM replicas are shown for the thermodynamic point with the highest density considered in this study. The GCM replicas were obtained within the five-variable dynamical model (34) and in general all three functions are equally well represented by the chosen dynamical model taking into account that no fitting parameters were used in construction of the GCM-replicas. Usually the time correlation functions of the dense liquid states are described by the generalized hydrodynamic theory worse than the dynamics of fluids with gas-like densities. Therefore we can accept the five-variable dynamical model as an appropriate one for analysis of the collective dynamics in a wide region of densities.

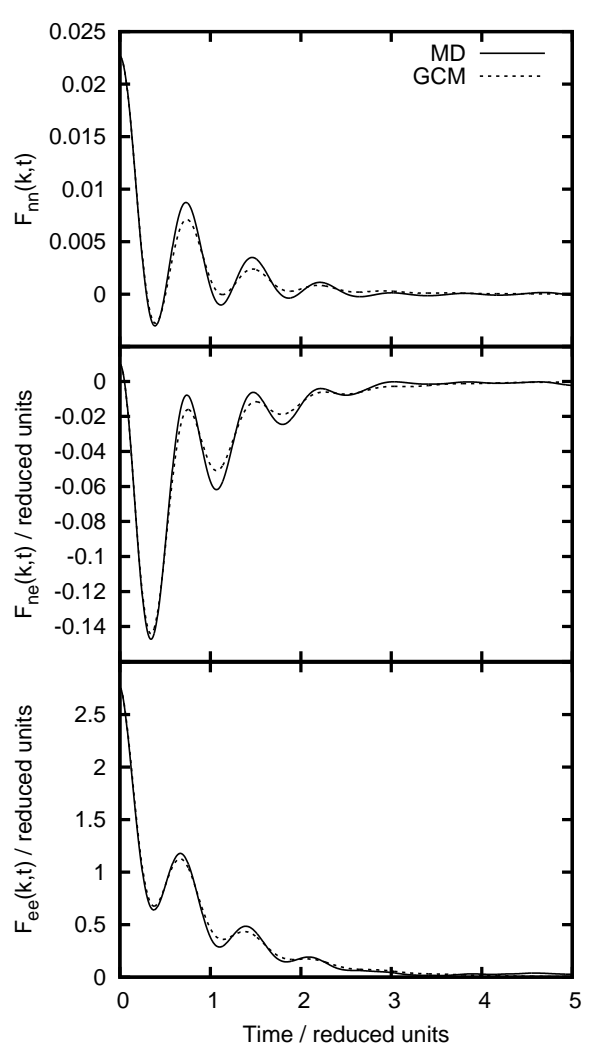

Figure 2. Density-density, density-energy and energy-energy time correlation functions for the density $n^{*}=1.011$ and temperature $T^{*}=1.71$ : by solid and dashed lines are shown MD-derived functions and their GCM replicas, respectively. Time and energy scales are $3.29 p s$ and $k_{\mathrm{B}} T$, respectively.

In this study we are mainly interested in modes connected with structural relaxation and relevant physical quantities. In figure 3 we show the values of adiabatic and high-frequency speed of sound estimated from the longwavelength asymptotes of MD-derived static averages $(\gamma(k) / S(k))^{1 / 2}$ and $\omega_{1}(k)$ [see equation 16 ], respectively. The lines in figure 3 correspond to a fit with a polynomial function $\left(a+b n^{2}\right)-$ in the wide region of density the obtained adiabatic and high-frequency speeds of sound were satisfactory fitted by such a dependence. The dependence of adiabatic and high-frequency speed of sound on density shown in figure 3 implies that the factor $\left(c_{\infty}^{2}-c_{\mathrm{s}}^{2}\right)$, which in the literature is sometimes called as the "structural relaxation strength" [15], increases with the density. Other factors, that define the behavior of the generalized relaxation process $d_{2}(k)$ on the macroscopic length scales (35) are the kinematic viscosity and ratio of specific heats. In figure 4 the generalized ratio of specific heats $\gamma(k)$ is presented for the thermodynamic points considered. This quantity was estimated using its definition [3] via the static heat density autocorrelations. This important generalized quantity reflects the static coupling between thermal and viscous processes on different length scales. On macroscopic distances, i.e. in the limit $k \rightarrow 0$, the functions $\gamma(k)$ tend to relevant constants, which decay with the density. This means, that the closer is the thermodynamic point to the solid phase the weaker will be static coupling between thermal and viscous processes. The generalized ratio of specific heats $\gamma(k)$ makes evidence, that for Lennard-Jones fluids one cannot neglect the coupling of density and thermal fluctuations. One can also see in figure 4 that the generalized ratio $\gamma(k)$ depends strongly on $k$, so that $\gamma$ cannot be taken in the generalized hydrodynamic approaches as a simple independent on wavenumber constant with its macroscopic value.

In figure 5 we show another important quantity, that defines the macroscopic lifetime of the kinetic relaxation process $d_{2}(k)$, the kinematic viscosity $D_{\mathrm{L}}$ as a function of density. The closed boxes in figure 5 represent the values obtained from the direct calculations of the Green-Kubo integrals [16], while the values shown by open boxes were extracted from the asymptotes of hydrodynamic modes taken at the smallest wavenumbers reached in MD simulations for each density. In general, the dependence of kinematic viscosity implies the divergence of $D_{\mathrm{L}}$ by approaching the 
freezing point, as it should be. Looking at the expression (35) for the kinetic relaxation process one can realize, that at the freezing point the relaxation time of this mode

$$
\tau_{2}(k \rightarrow 0)=\frac{D_{\mathrm{L}}}{c_{\infty}^{2}-c_{\mathrm{S}}^{2}}
$$

becomes infinite, that is a feature of the structural relaxation, and namely the alpha-relaxation with macroscopically large relaxation time. Hence, two facts, discussed above, support the rele-

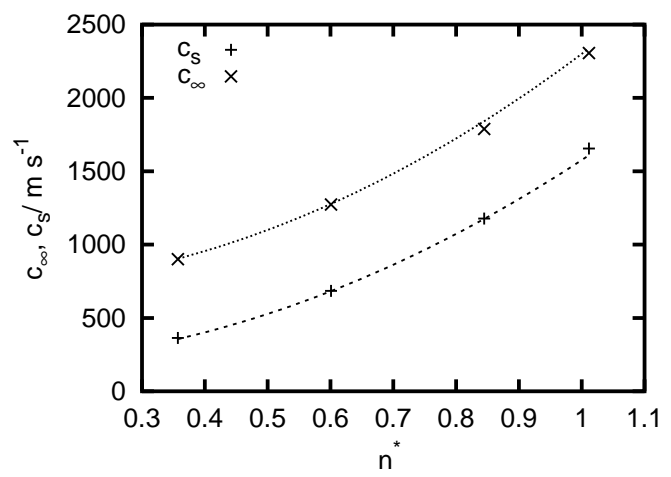

Figure 3. Infinite and adiabatic speed of sound along the isothermal line $T^{*}=1.71$ for four different densities of Lennard-Jones fluids. The lines are a fit with $a+b n^{2}$ function.

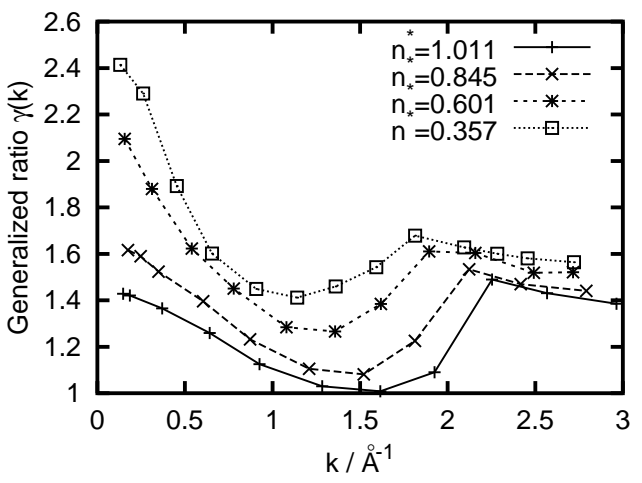

Figure 4. Generalized ratio of specific heats $\gamma(k)$ as functions of wavenumber for different density of Lennard-Jones fluid at $T^{*}=$ 1.71 .

vance of the kinetic relaxation mode $d_{2}(k)$ to the structural relaxation, namely, the presence of the factor called "strength of structural relaxation" and divergence of the relaxation time at the freezing point. Another interesting consequence follows from the dependence of the relaxation mode $d_{2}(k)$ on kinematic viscosity (35). In fact this mode restricts the region of wavenumbers, where hydrodynamic collective modes behave strictly according to the hydrodynamic expressions. The larger is kinematic viscosity the smaller will be the window of hydrodynamic regime (see [22] for analytical expressions).

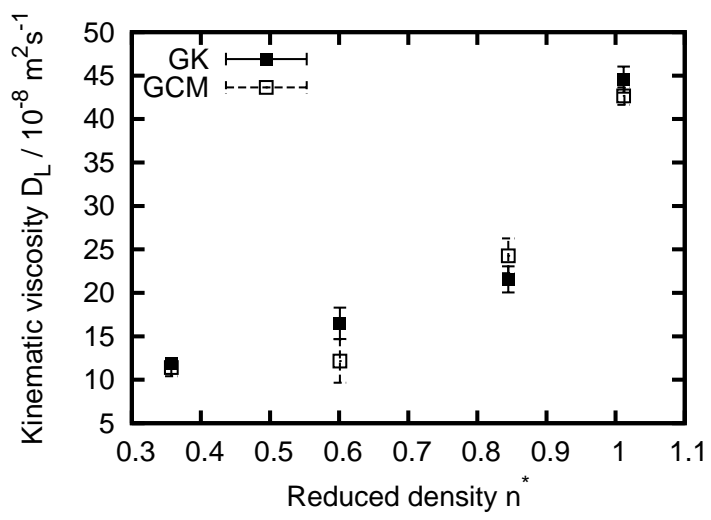

Figure 5. Kinematic viscosity as function of density at $T^{*}=1.71$. Calculations of Green-Kubo integrals (GK) and values estimated from long-wavelength asymptotes of generalized modes (GCM) are shown by plus and cross symbols with errorbars, respectively.

The propagating $z_{j}(k)$ and relaxing $d_{j}(k)$ dynamical eigenmodes in the wide region of wavenumbers for the thermodynamic point with the highest density considered in this study are shown in figure 6 . For the chosen five-variable dynamical modes we obtained five eigenvalues for each $k$-point 
sampled in MD simulations. In the long-wavelength region we have obtained as the eigenvalues one complex-conjugated pair and three real numbers. Looking at their wavenumber-dependence and using the asymptotes of hydrodynamic modes with the values $c_{\mathrm{S}}, \gamma, D_{\mathrm{L}}, D_{\mathrm{T}}$, estimated beyond the GCM framework as in figures 3 and 4 and from Green-Kubo integrals, one can see in figure 6 a nice agreement with the quadratic dependence on $k$ of the sound damping $\operatorname{Re} z_{\mathrm{s}}(k)$ and thermal diffusivity mode $d_{\mathrm{T}}(k)$, while the dispersion $\operatorname{Im} z_{\mathrm{s}}(k)$ of acoustic excitations has a positive deviation called "positive dispersion". We show by dashed lines the expected hydrodynamic linear dispersion law $c_{\mathrm{s}} k$ and the dispersion law given by equation (29) obtained within a simplified three-variable model, which actually describes local coupling effects between acoustic excitations and structural relaxation. One can see that the analytical expression equation (29) correctly reproduces the positive deviation in the small-wavenumber region.

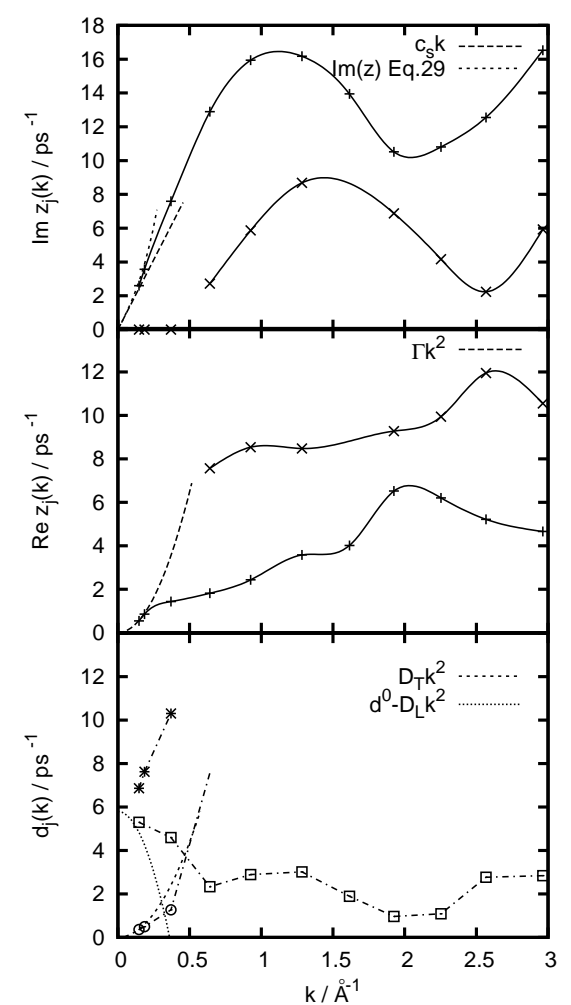

Figure 6. Imaginary and real parts of generalized collective modes for the density $n^{*}=1.011$ and temperature $T^{*}=1.71$. For convenience the purely real eigenvalues are noted as $d_{j}(k)$ and shown in the bottom frame. Propagating modes are shown by symbols connected by solid line and relaxation modes are represented by symbols connected by dash-dotted line. By dashed lines in the upper frame the linear dispersion law with adiabatic speed of sound and positive dispersion of sound branch that follows from equation (29) are shown. Dashed line in middle frame shows quadratic long-wavelength asymptotes of sound damping, and in the bottom frame - thermal relaxation mode. The dotted line in the bottom frame corresponds to the analytical result for the structural relaxation without coupling to thermal processes represented by equation (27).
In the region $k>0.6 \AA^{-1}$ there appears another branch of propagating excitations, which corresponds to kinetic propagating heat excitations (heat waves). We stress that the majority of the generalized hydrodynamic studies of collective dynamics in liquids usually restrict treatment of the thermal fluctuations just by the most slow ones, while correct treatment requires to account for the same level of short-time processes in viscous and thermal description of dynamics. The heat waves appear in complete analogy with shear waves, which are not supported by liquid systems on macroscopic distances. For more detailed information on heat waves and convergence of relevant eigenmodes with increasing number of dynamical variables we refer to [23]. Hence, the GCM approach predicts, that the thermal relaxation processes on the nanoscale in Lennard-Jones liquid are unstable with respect to emergence of propagating heat excitations, which can contribute to heat transport at the nanoscale. For the generalized mode treatment this means, that instead of two real eigenmodes shown in bottom frame of figure 6 by open circles and asterisks one obtains for $k>0.6 \AA^{-1}$ another pair of complex-conjugated eigenvalues with the frequency smaller than of the generalized acoustic excitations (high-frequency branch in the top frame of figure 6 ).

The most interesting is the behavior of relaxation process $d_{2}(k)$ as the function of the wavenumber (open boxes connected by dash-dotted line in the bottom frame of figure 6$)$. The predicted by the analytical model wavenumber dependence (27) shown in figure 6 by dotted line implies, that the lifetime of this relaxation process becomes larger with increasing $k$ and beyond the hydrodynamic region the mode $d_{2}(k)$ becomes 
smaller than $d_{\mathrm{T}}(k)$, which though having small negative deviation tends to larger values. The coupling with thermal processes according to (35) increases the $d_{2}(k)$, that is in agreement with numerical results. But the most interesting fact is that the mode $d_{2}(k)$, connected with structural relaxation, becomes the main relaxation process in the region of intermediate and large wavenumbers. One can make additional checks with different subsets of dynamical variables as performed in $[23,24]$ in order to discard any relation between the mode $d_{2}(k)$ and thermal processes in that range of wavenumbers.

The wavenumber dependence of hydrodynamic relaxation and propagating modes in the wide $k$-region is tightly connected with generalized transport coefficients. For example, the negative deviation of the thermal relaxation mode $d_{\mathrm{T}}(k)$ can be associated with decreasing of generalized wavenumber-dependent thermal diffusivity $D_{\mathrm{T}}(k)$. The most correct and transparent way of introduction of the generalized transport coefficients is via the matrix of lowest order (or hydrodynamic) memory functions $[25,26]$. For the case of pure liquids the $3 \times 3$ hydrodynamic matrix of lowest memory functions is directly connected to the generalized transport coefficients $\tilde{\mathscr{L}}_{i j}(k, \omega)$ :

$$
\tilde{\mathbf{M}}(k, \omega)=k^{2} V k_{B} T \tilde{\mathscr{L}}(k, \omega) \mathbf{F}(k, t=0),
$$

where $\tilde{\mathscr{L}}(k, \omega)$ is the $3 \times 3$ matrix of generalized wavenumber- and frequency-dependent transport coefficients, and $V$ is the volume of the system. As an example we show in figure 7 the generalized static kinematic viscosity $D_{\mathrm{L}}(k, \omega=0)$ and thermal diffusivity $D_{\mathrm{T}}(k, \omega=0)$. The general tendency is the monotonic decay of these quantities with increasing wavenumber.

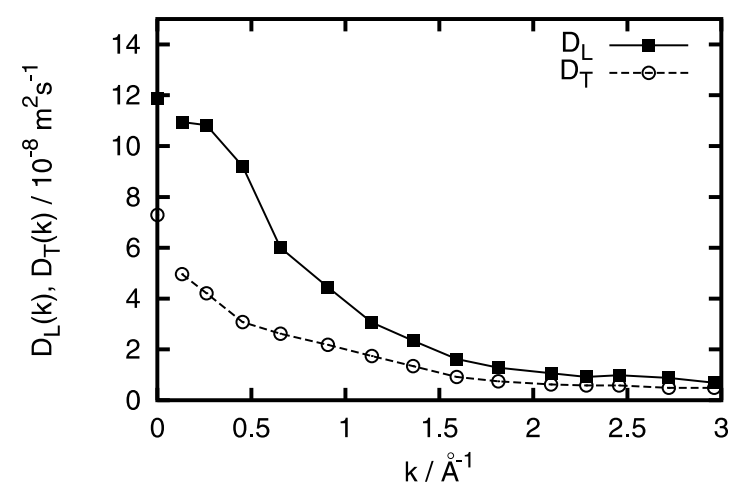

Figure 7. Generalized wavenumber-dependent kinematic viscosity $D_{\mathrm{L}}(k)$ and thermal diffusivity $D_{\mathrm{T}}(k)$ for $n^{*}=0.357$. At $k=0$ the values of relevant Green-Kubo integrals are shown.

The most important issue in the study of non-hydrodynamic processes in liquids is the contribution from the kinetic processes to the observable dynamical structure factor $S(k, \omega)$, or its time-Fourier counterpart the density-density time correlation function. In figure 8 we show the mode contributions to the $F_{n n}(k, t) / S(k)$ represented in the form suggested in [24]. Hydrodynamic theory predicts, that for pure fluids there exist only two contributions to the density-density time correlation functions: from the thermal diffusivity and sound excitations with the weights $(\gamma-1) / \gamma$ and $1 / \gamma$, respectively. In figure 8 at $k=0$ the corresponding contributions are shown by open and closed circles. The GCM approach predicts, that when $k$ increases there appear additional contributions from the kinetic processes and all the weight coefficients must depend strongly on $k$. One can see in figure 8, that the contribution from generalized acoustic excitations and thermal relaxation mode are decaying with the increasing wavenumber, while the corresponding contribution from the kinetic relaxation process $d_{2}(k)$ increases and becomes dominant for representing the relaxational behavior of $F_{n n}(k, t) / S(k)$. The kinetic heat waves almost do not contribute to the shape of $F_{n n}(k, t) / S(k)$. The increase of the lifetime of the process $d_{2}(k)$ and its contribution to the density-density time correlation function in the region $k \approx 2.0-2.2 \AA^{-1}$, where the main maximum of the static structure factor is located, means that the structural relaxation mode $d_{2}(k)$ is responsible for the increasing of the long-lasting tails of the density-density time correlation functions and corresponding narrowing of the central peak of $S(k, \omega)$ known as the de Gennes narrowing [2]. 


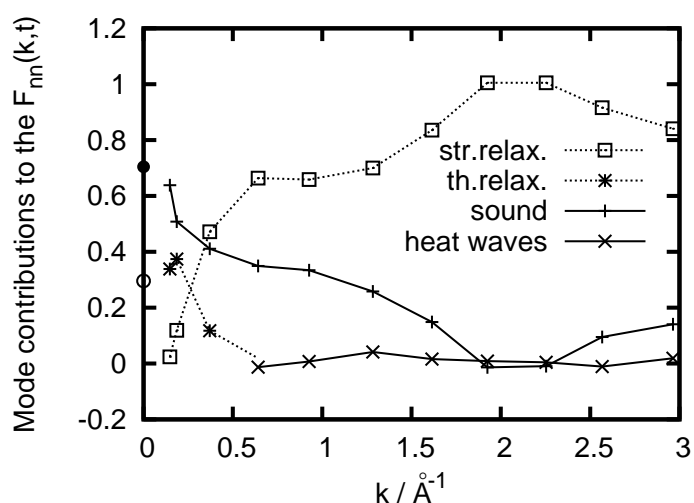

Figure 8. Wavenumber-dependent mode contributions to the density-density time correlation functions from different propagating and relaxation collective modes for the density $n^{*}=1.011$ and temperature $T^{*}=1.71$. The closed and open circles at $k=0$ show predicted by hydrodynamics values for contributions from sound excitations and thermal relaxation mode, respectively.

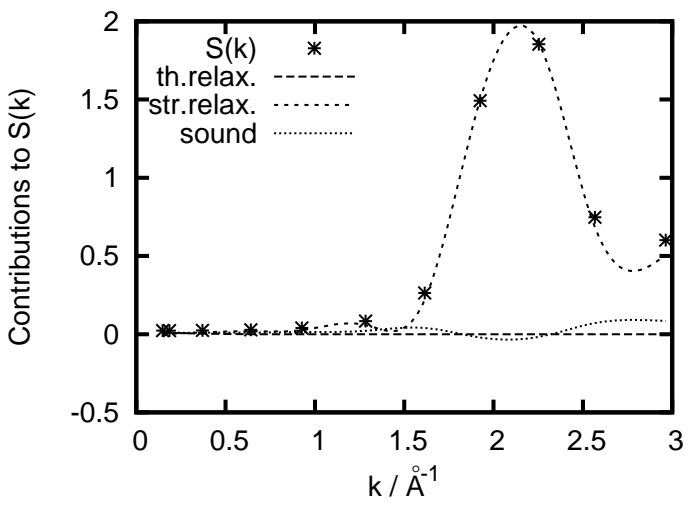

Figure 9. Zero-time contributions from generalized collective modes to the shape of static structure factor $S(k)$. Leading contribution to the shape of $S(k)$ from the slow mode shown by spline interpolated short-dashed line proves its relation to the wavenumberdependent structural relaxation.

Formally one can use the mode contributions to the density-density time correlation function $F_{n n}(k, t)$ and represent them at $t=0$ as the contributions to the static structure factor $S(k)$ from different collective processes. Intuitively this means, that each instantaneous configuration one can represent as some deviations from a reference configuration due to sound propagation, structural relaxation, etc. In figure 9 we show, that the contributions from generalized modes to $F_{n n}(k, t)$ at $t=0$ have the only leading term, which comes from the relaxation mode $d_{2}(k)$, that once more proves its origin as the mode of structural relaxation, because it is directly related to the main structural quantity of the system - its structure factor.

\section{Conclusions}

In this study we applied the approach of Generalized Collective Modes to estimation of wavenumber dependence of structural relaxation in pure liquids. The GCM approach has several advantages over the standard memory function treatment of generalized hydrodynamics. In particular, it represents the contributions to the time correlation functions from hydrodynamic and non-hydrodynamic processes via exponential functions of time thus making very transparent estimation of relaxation time for non-hydrodynamic relaxation processes. In contrast to the standard memory function treatment of structural relaxation on introducing additional decay channels in the second order memory function, the GCM approach systematically takes into account non-hydrodynamic processes via additional non-hydrodynamic variables allowing to estimate the relevant combinations for each non-hydrodynamic collective mode in different regions of wavenumbers.

In this study we have shown, how using the GCM approach one can separate a contribution from the structural relaxation to the density-density time correlation functions in a wide range of wavenumbers and estimate the wavenumber dependence of the corresponding relaxation time. The GCM approach predicts several features in the wavenumber dependence of structural relaxation in pure liquids, namely, it is found that:

(i) in the long-wavelength limit the structural relaxation tends to a finite relaxation time defined by the kinematic viscosity and a factor called in the literature as "strength of structural relaxation" [15];

(ii) at small wavenumbers this relaxation time increases, being in opposite tendency with hydro- 
dynamic thermal relaxation process with decreasing relaxation time;

(iii) by decreasing temperature or increasing density the width of hydrodynamic region, i.e. the wavenumber range where collective modes behave strictly according to the hydrodynamic expressions, becomes more narrow and the non-hydrodynamic excitations play essential role in wider range of $k$;

(iv) for intermediate and large wavenumbers the structural relaxation almost completely defines the shape of the central peak of dynamical structure factor, while thermal processes do not have much effect on the central peak. The relevance of the slow relaxation process at intermediate and large wavenumbers to the structural relaxation is demonstrated via its contribution for the instantaneous configurations to the structure factor $S(k)$.

This study was aimed to estimate the role of non-hydrodynamic process of structural relaxation in collective dynamics of pure liquids and to look at the effect of liquid density on different parameters, that define the structural relaxation in liquids. The study of temperature dependence of structural relaxation within the GCM approach will be reported elsewhere.

\section{References}

1. March N.H., Tosi M.P. Atomic Dynamics in Liquids. London, Macmillan Press, 1976.

2. Hansen J.-P., McDonald I.R. Theory of Simple Liquids. London, Academic, 1986.

3. de Schepper I.M., Cohen E.G.D., Bruin C., van Rijs J.C., Montfrooij W., de Graaf L.A., Phys. Rev. A, 1988, 38, 271.

4. Cohen C., Sutherland J.W.H., Deutch J.M., Phys. Chem. Liq., 1971, 2, 213.

5. Akcasu A.Z., Daniels E., Phys. Rev. A, 1970, 2, 962.

6. Tong E., Desai R.C., Phys. Rev. A, 1970, 2, 2129.

7. Ailawadi N.K., Rahman A., Zwanzig R., Phys. Rev. A, 1971, 4, 116.

8. Suck J.-B., J. Neutr. Res., 2006, 14, 317.

9. Mezei F., Neutron scattering and collective dynamics in liquids and glasses - In: Liquids, Freezing and Glass Transition. Eds. Hansen J.-P., Levesque D., J.Zinn-Justin. Elsevier, 1991.

10. Boon J.-P., Yip S. Molecular Hydrodynamics. New-York, McGraw-Hill, 1980.

11. Scopigno T., Ruocco G., Sette F., Rev. Mod. Phys., 2005, 77, 881.

12. Scopigno T., Balucani U., Ruocco G., Sette F., J. Phys.:Cond. Matt., 2000, 12, 8009.

13. Scopigno T., Balucani U., Ruocco G., Sette F., Phys. Rev. Lett., 2000, 85, 4076.

14. Bencivenga F., Cunsolo A., Krisch M., Monaco G., Ruocco G., Sette F., Europhys. Lett., 2006, 75, 70 .

15. Bencivenga F., Cunsolo A., Krisch M., Monaco G., Orsingher L., Ruocco G., Sette F., Vispa A., Phys. Rev. Lett., 2007, 98, 085501.

16. Balucani U., Zoppi M. Dynamics of the Liquid State. Oxford, Clarendon Press, 1994.

17. Omelyan I.P., Tokarchuk M.V., J. Phys.: Condens. Matt., 2000, 12, L505.

18. Mryglod I.M., Omelyan I.P., Tokarchuk M.V., Mol. Phys., 1995, 84, 235.

19. Bryk T., Mryglod I., Low Temp. Phys., 2007, 33, 790.

20. Bryk T., Mryglod I., J. Phys.: Cond. Matt., 2005, 17, 413.

21. Bryk T., Mryglod I., Condens. Matter Phys., 2007, 10, 481.

22. Bryk T., Mryglod I., Condens. Matter Phys., 2004, 7, 471.

23. Bryk T., Mryglod I., Phys. Rev. E, 2001, 63, 051202.

24. Bryk T., Mryglod I., Phys. Rev. E, 2001, 64, 032201.

25. Mryglod I.M., Omelyan I.P., Mol. Phys., 1997, 92, 913.

26. Bryk T., Mryglod I., Condens. Matter Phys., 2004, 7, 285. 


\title{
Структурна релаксація у простих рідинах: аналіз залежності від хвильового числа в рамках підходу узагальнених колективних мод
}

\author{
Т.Брик ${ }^{1,2}$, І.Мриглод ${ }^{1,2}$ \\ 1 Інститут фізики конденсованих систем НАН України, 79011 Львів, Україна \\ 2 Інститут прикладної математики та фундаментальних наук, Національний університет “Львівська \\ політехніка", 79013 Львів, Україна
}

Отримано 4 січня 2008 р., в остаточному вигляді - 28 лютого 2008 р.

\begin{abstract}
Залежність від хвильового числа для структурної релаксації в рідинах досліджується методом узагальнених колективних мод (УКМ). Запропоновано новий пертурбативний підхід в рамках методу УКМ, що застосовано для знаходження залежної від хвильового числа структурної релаксації та дисперсії звуку у довгохвильовій границі в рамках спрощеної тризмінної динамічної моделі. Аналітичні результати порівнюються із числовими дослідженнями узагальнених мод в рамках більш загальної п'ятизмінної динамічної моделі, що враховує термічні процеси в рідинах. Представлено числові результати для чотирьох термодинамічних точок ленард-джонсівського плину з приведеною температурою $T^{*}=1.71$. Обговорюються особливості негідродинамічного процесу структурної релаксації у різних областях хвильових чисел.
\end{abstract}

Ключові слова: узагальнена гідродинаміка, структурна релаксація, динамічні моделі, звукові збудження, позитивна дисперсія

PACS: 05.20.Jj, 61.20.LC, 62.60.+V 